\title{
Water Absorption Behaviour in Polyethylene Boron Nitride Nanocomposites
}

\author{
R. Ayoob, T. Andritsch, A. S. Vaughan \\ Tony Davies High Voltage Laboratory \\ University of Southampton \\ Southampton, UK \\ ra1e10@ecs.soton.ac.uk
}

\begin{abstract}
Polymer nanocomposites, when prepared and treated carefully, have the potential to offer many advantages over the current materials used in high voltage applications. Four materials have been prepared containing $2 \mathrm{wt} \%, 5 \mathrm{wt} \%$, and 10 wt\% of hexagonal boron nitride nanofiller, including an unfilled material based on a polyethylene blend. The water absorption behavior was studied by measuring the change in mass in the different samples, at different humidity conditions, and using dielectric spectroscopy. Dielectric spectroscopy was used to measure both the real relative permittivity and the dielectric losses. Under dry and ambient conditions, the materials behave similarly and exhibit insignificant losses. Under wet conditions, the unfilled polyethylene does not seem to be affected, however, the nanocomposites exhibit some dielectric losses as expected. The wet samples were easily dried off and therefore behaved similarly to the originally dry samples with no apparent losses.
\end{abstract}

Keywords-nanocomposites; dielectric spectroscopy; water absorption; high voltage

\section{INTRODUCTION}

Polymer nanocomposites are a class of materials in which a filler, with dimensions of $100 \mathrm{~nm}$ or less, is incorporated into a polymer matrix which brings about new material properties [1]. There has been a global interest in these materials as they can potentially enhance electrical properties such as improved breakdown strength, reduced space charge accumulation, and lower dielectric losses. However, a lot of variability exists in the literature regarding the electrical performance of polymer nanocomposites. Possible reasons for the existing inconsistencies could be due to different material preparation techniques, nanoparticle agglomeration, unknown filler content, inconsistent sample storage conditions, and unknown water level content in the samples. Although issues such as agglomeration may be inevitable, others, such as the sample water level content can be easily solved by studying how the material behaves under different conditions. Since many thermoplastics such as polyethylene and polypropylene are hydrophobic, this isn't necessarily true with the type of nanofiller used as most of them contain hydroxyl groups on their surface, which can promote water absorption [2].

In this study a polymer nanocomposite system composed of a polyethylene blend as the host polymer and hexagonal boron nitride (hBN) as the filler is used. Unlike most spherical nanofillers which contain hydroxyl groups surrounding their surface, hexagonal boron nitride contains some hydroxyl groups attached to the edges of its stacked platelets rather than on its surface thus rendering it more hydrophobic than other types of fillers [3]. Since many studies have shown the effect of water absorption on hydrophilic filler, this study therefore explores the importance of surface chemistry by studying how water affects nanocomposites containing hydrophobic fillers.

\section{EXPERIMENTAL}

\section{A. Sample Preparation}

The host polymer in this study is a polyethylene blend composed of $80 \%$ LDPE, grade LD100BW obtained from ExxonMobil Chemicals, and 20\% HDPE, grade Rigidex HD5813EA obtained from BP Chemicals. The hexagonal boron nitride powder was grade NX-1 obtained from Momentive with a quoted particle size of $900 \mathrm{~nm}$. In all cases, a total of $10 \mathrm{~g}$ of polymer was used. The polymer was added to $100 \mathrm{~mL}$ of xylene and the solution was heated to the boiling point of xylene. The polymer solution was then stirred with a magnetic stirrer at the boiling point of xylene until all the polymer had dissolved. The solution was then taken off the heat and stirred using a mixing rod until it slightly thickened. The required amount of $\mathrm{hBN}$ was dispersed in isopropyl alcohol (IPA). This $\mathrm{hBN}$ suspension was then added to the polymer solution and stirred continuously until a waxy solid has formed. The resultant material was left in a fume cupboard for $7 \mathrm{~d}$ followed by drying in a vacuum oven for $3 \mathrm{~d}$ at $60{ }^{\circ} \mathrm{C}$ to remove any residual solvent. The required samples were then prepared by melt pressing at a temperature of $180{ }^{\circ} \mathrm{C}$ with a 5 ton load. A total of four materials were prepared containing 0 $\mathrm{wt} \%, 2 \mathrm{wt} \%, 5 \mathrm{wt} \%$, and $10 \mathrm{wt} \%$ of filler. The materials are abbreviated as PEBN/X, e.g. PEBN/5 refers to a polyethylene boron nitride nanocomposite with $5 \mathrm{wt} \%$ of boron nitride.

\section{B. Sample Conditioning}

After all the samples have been prepared, they were exposed to the laboratory ambient conditions $\left(20 \pm 2{ }^{\circ} \mathrm{C}\right.$ and 50 $\pm 20 \% \mathrm{RH}$ ) for $14 \mathrm{~d}$ to allow the mass of each sample to reach equilibrium. The samples were then split into four different groups termed: "Ambient" (exposed to ambient conditions for a further $14 \mathrm{~d}$ ), "Dry" (dried in vacuum at room temperature for $14 \mathrm{~d}$ ), "Wet" (immersed in water for $14 \mathrm{~d}$ ), and "Wet to 
Dry" (dried in vacuum oven for $14 \mathrm{~d}$ after being immersed in water for $14 \mathrm{~d}$ ). The mass of the samples was recorded before and after the appropriate conditioning to understand how much water the material absorbs.

\section{Dielectric Spectroscopy}

Dielectric spectroscopy was used to study the dieletric response. A Solartron 1296 dielectric interface with a Schlumberger SI 1260 impedance/phase gain analyser system was used to perform the measurements. An AC voltage of $1 \mathrm{~V}$ was applied with a frequency sweep from $0.1 \mathrm{~Hz}$ to $1 \mathrm{MHz}$. Samples $250 \mu \mathrm{m}$ in thickness were prepared and gold coated before, finally, being tested between $30 \mathrm{~mm}$ diameter electrodes.

\section{RESULTS AND DISCUSSION}

Table I shows the mass changes recorded in all of the samples after all types of conditioning. The figures shown are compared to the initial mass of the sample before conditioning, that is after being exposed to ambient conditions. In the case of the Ambient and Dry samples, the mass apparently fluctuated around a single point throughout the $14 \mathrm{~d}$, with no systematic variation. The Wet samples reached saturation approximately 3 $\mathrm{d}$ after being immersed in water. After $14 \mathrm{~d}$ in water, all the composites exhibited a small mass change of less than $0.05 \%$, that is they did not absorb a great deal of water. In the case of the Wet to Dry samples, the final mass was compared to the initial mass before being immersed in water. The mass changes, which is less than $0.1 \%$, suggests that the mass of these samples has decreased and returned to around the initial mass thus losing all the absorbed water.

Dielectric spectroscopy measurements of the real relative permittivity and the dielectric loss tangent of the Dry samples are shown in Fig. 1. Although measurements were taken on different days over a $14 \mathrm{~d}$ period, only the data acquired on the $14^{\text {th }}$ day are shown, since no differences were observed. The figure shows that the real relative permittivity is frequency independent for all the materials. The value of the real permittivity appears to increase with increasing filler content. However, the addition of boron nitride, even in the $10 \mathrm{wt} \%$ composite, does not seem to have a significant effect on the permittivity compared to the value of the unfilled sample. The dielectric loss tangent measurements show that all the materials behave similarly and have almost no losses. The values of the measured losses, which is much less than 0.01 for all materials, fall within the noise limit of the equipment and, thus it can be said that the materials show no dielectric losses when fully dry.

TABLE I. MASS CHANGES AFTER CONDITIONING

\begin{tabular}{|c|c|c|c|c|}
\hline \multirow{2}{*}{ Material } & \multicolumn{4}{|c|}{ Mass changes after conditioning } \\
\cline { 2 - 5 } & Ambient & Dry & Wet & Wet to Dry \\
\hline PEBN/0 & $<0.01 \%$ & $<0.01 \%$ & $<0.01 \%$ & $<0.01 \%$ \\
\hline PEBN/2 & $<0.01 \%$ & $<0.01 \%$ & $+0.0303 \%$ & $<0.01 \%$ \\
\hline PEBN/5 & $<0.01 \%$ & $<0.01 \%$ & $+0.0405 \%$ & $<0.01 \%$ \\
\hline PEBN/10 & $<0.01 \%$ & $<0.01 \%$ & $+0.0493 \%$ & $<0.01 \%$ \\
\hline
\end{tabular}

It is important to note that this scale was chosen so as to have the same scale in Fig. 3, for comparison purposes.

Fig. 2 shows dielectric spectroscopy data obtained from the Ambient samples. The behaviour of the Ambient and Dry samples almost identical, cf. Fig. 1 and Fig. 2. The real relative permittivity shows the same trend, with the same values. The dielectric loss tangent is also very minimal (much less than $0.01)$ as in the case of the Dry samples. This is expected, since the mass of the ambient samples did not change over the $14 \mathrm{~d}$ so the samples did not absorb any water suggesting they are already dry. Since very few hydroxyl groups are available on the surface of $\mathrm{hBN}$, they are unlikely able to absorb water from ambient conditions. Since the ambient conditions with humidity levels of up to $60 \%$ did not affect the behaviour of these materials, the next step was to directly immerse the samples in water to determine whether or not they will absorb water under those conditions.

Fig. 3 shows the dielectric response of the samples that have been immersed in water for $14 \mathrm{~d}$. A noticeable difference can be observed in the behaviour of the Wet nanocomposites samples compared to the Dry and Ambient samples. In contrast the unfilled polyethylene does not seem to be affected by water immersion. As the filler loading increases, the amount of absorbed water increases, so increasing the permittivity. The real relative permittivity is frequency dependent in this case and appears to increase as the frequency is lowered with all nanocomposites. Since there is some water effects across the entire frequency range, this suggests that different modes of water exist in the system as the permittivity of water is very high compared to these nanocomposites so small amounts of water will influence the overall permittivity due to polarization. In the case of the dry PEBN/10 sample, the real relative permittivity increases from 2.52 to $2.54(<1 \%$ increase) as the frequency is decreased. In the Wet PEBN/10 sample, the real relative permittivity increases from 2.58 to 2.96 (15\% increase) as the frequency is decreased. Although this sample absorbed $0.05 \%$ of water, it had a considerable effect on the real relative permittivity.

The value of the dielectric loss tangent increases with increasing at filler, over the entire measured frequency range. This is again consistent with the real permittivity data confirming the existence of water in a range of different states. When the dielectric loss tangent behaviour of the wet samples is considered, it might seem that there is some sort of broad loss peak present in all of the nanocomposites in the frequency region between $10^{4}$ and $10^{5} \mathrm{~Hz}$. This could be explained as an effect due to the presence of water which can be polarized at those frequencies. However, a closer look at Fig. 3 b shows that this "peak" that is observed at those frequencies is present at the exactly same frequency in all the samples, including the unfilled polyethylene which does not exhibit any losses. The same "peak" is observed in Fig. 1 b, Fig. 2 b, and Fig. 4 b in which no water has been absorbed suggesting that this effect might be an artefact of the equipment instead of a loss peak due to the presence of water. As the filler content increases, the value of loss tangent, which is almost frequency independent, increases in turn due the larger amount of absorbed water. However, since the value of the loss tangent is less than 0.02 in all samples, it is a very insignificant loss for a nanocomposite. 

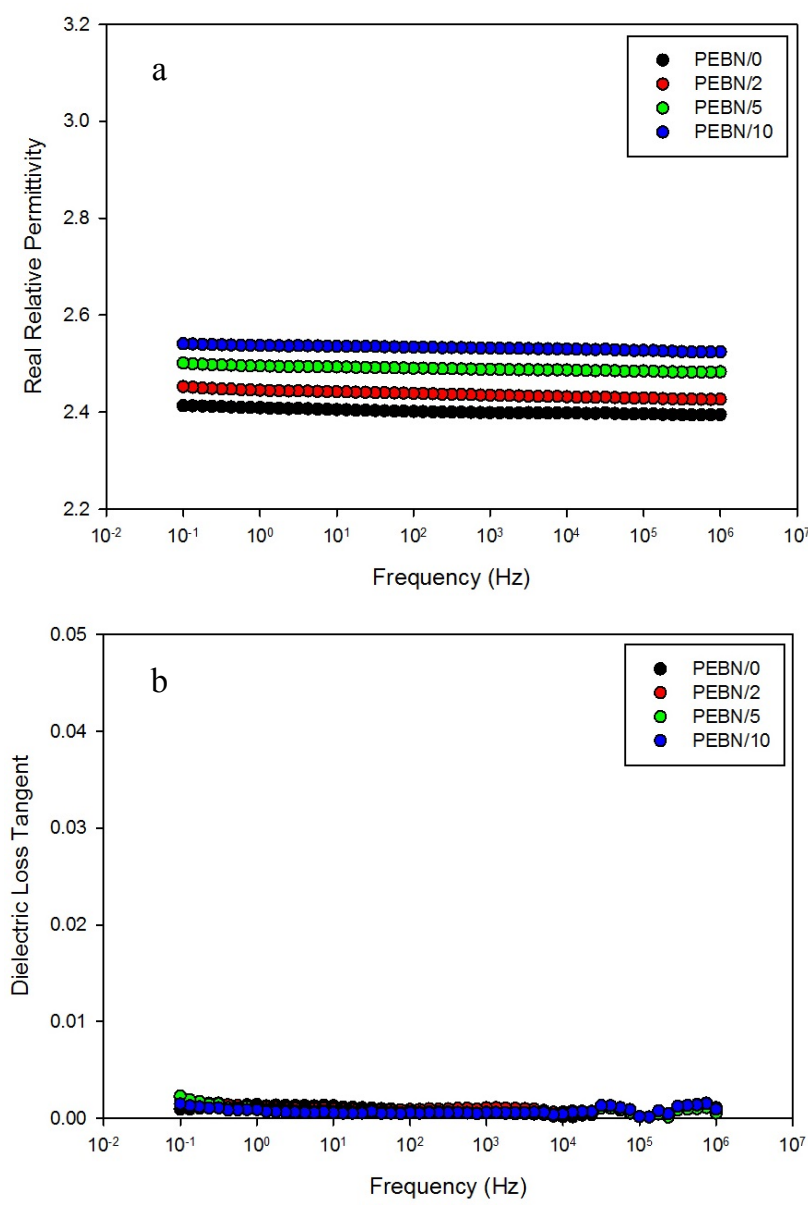

Fig. 1. Dielectric spectroscopy measurements showing (a) real relative permittivity, (b) dielectric loss tangent of the dry samples

If water is tightly bound to the surface, this might be evident in the loss tangent measurements as a loss peak at low frequencies whereas this peak will be present at higher frequencies if the water is loosely bound. In order to understand the state of the present water, an effort has been to dry the Wet samples to investigate whether the water is tightly or loosely bound. The wet samples have been placed in vacuum for $14 \mathrm{~d}$ prior to testing. Fig. 4 shows the dielectric response of the wet samples after they have been dried. It is clear from Fig. 4 that the behaviour of these samples is again identical to the behaviour of the Dry and Ambient samples. The real relative permittivity has again become frequency independent and the values reduced to the same values in the dry and ambient samples. Similarly, the values of the loss tangent have decreased and are much less than 0.01 . Furthermore, the mass of the samples after they have been dried have returned to their initial mass before they were immersed in water. Dielectric spectroscopy measurements along with measurements in mass changes indicate that all the absorbed was completely dried off when the wet samples have been placed in vacuum. These results suggest that the water present in this system is free water that is loosely bound to the surface which was easily eliminated after the samples have been dried in vacuum. Further studies with mass monitoring have shown that the wet samples also lose all of their water
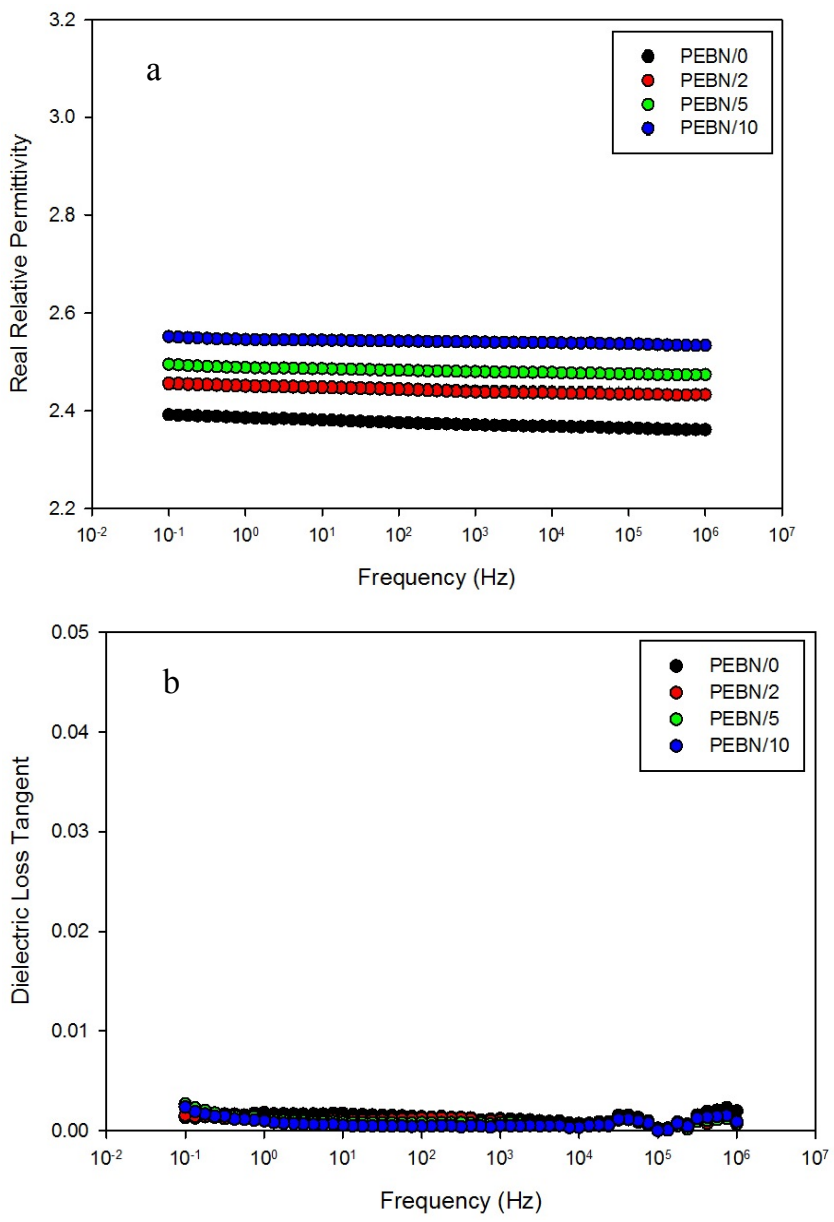

Fig. 2. Dielectric spectroscopy measurements showing (a) real relative permittivity, (b) dielectric loss tangent of the ambient samples

content when exposed to ambient conditions, not just vacuum, confirming that the water is not tightly bound.

Not many studies have been done on the effect of water absorption in boron nitride based nanocomposites due to their hydrophobic nature. Unlike the systems shown in this study, silica based nanocomposites have been found to have double relaxations showing two distinct loss peaks, one corresponding to loosely bound water and the other to tightly bound water. The loss peaks move to higher frequencies with increasing amounts of absorbed water [4-6]. A study has been done on nanocomposites with a similar platelet like structure as boron nitride in which mid frequency relaxation peaks were observed that are attributed to presence of water [7]. In this study, no similar relaxation features are observed as the water is present in many different states in the boron nitride, which in turn causes the loss tangent to increase in all frequencies. In another study, a successful attempt has been made to completely remove the hydroxyl groups from the surface of silica by calcination, turning the silica from a hydrophilic into a hydrophobic state, thus emphasizing the importance of the surface chemistry of the fillers when using them in polymer nanocomposites [8]. The surface chemistry of $\mathrm{hBN}$ prevents water absorption, unlike other fillers which enhance water absorption. 

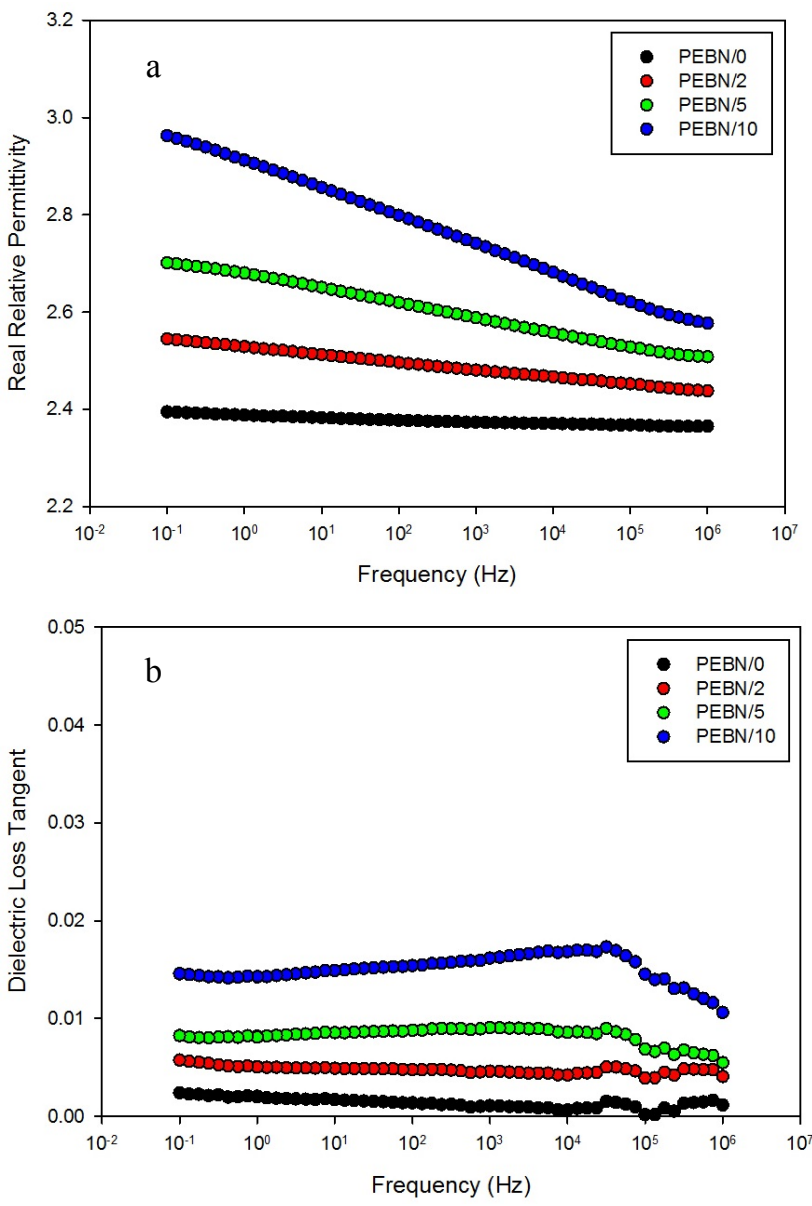

Fig. 3. Dielectric spectroscopy measurements showing (a) real relative permittivity, (b) dielectric loss tangent of the wet samples

\section{CONCLUSION}

The above results show that the real relative permittivity and the dielectric loss tangent of the Dry and Ambient samples behave exactly the same. The real relative permittivity increases with increasing filler content and is frequency independent. The dielectric loss tangent is very small and insignificant suggesting no losses in the nanocomposites.

Unlike other fillers in literature such as silica or silicon nitride, which absorb water under ambient conditions, hBN only absorbs water when directly immersed in water. Dielectric spectroscopy measurements showed that the nanocomposites absorb an insignificant amount of water, however, it is clearly reflected in the permittivity and loss tangent of the materials in which both increase with increasing filler content over all measured frequencies. Although they absorb some water, it can be completely dried off when the Wet samples are placed under vacuum or ambient conditions. As long the nanocomposites are not directly immersed in water, they will not absorb any moisture and will not exhibit any losses. The lack of available surface hydroxyl groups in hBN makes it a perfect type of filler to use in polymer nanocomposites. With proper conditioning and treatment, polyethylene $\mathrm{hBN}$ nanocomposites are promising materials for use in future high voltage applications.
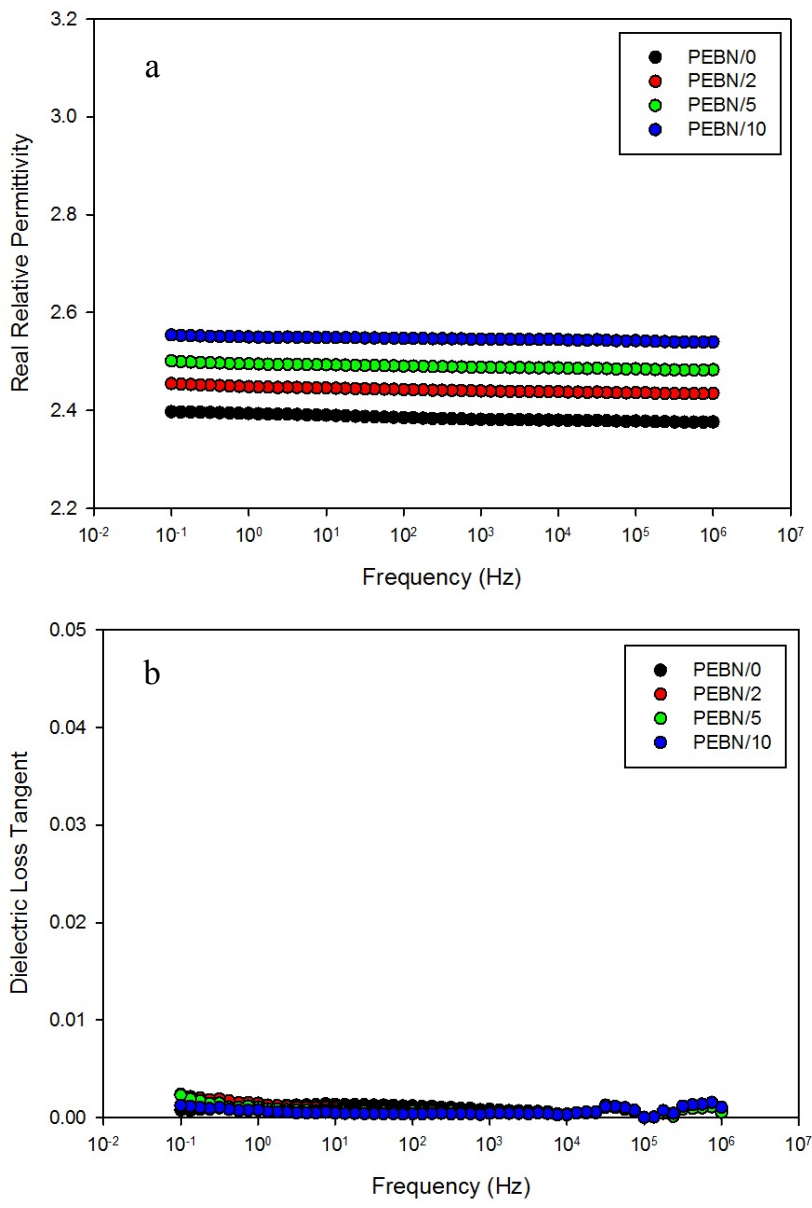

Fig. 4. Dielectric spectroscopy measurements showing (a) real relative permittivity, (b) dielectric loss tangent of the wet to dry samples

\section{REFERENCES}

[1] T. J. Lewis, "Nanometric dielectrics," IEEE Trans. Dielectr. Electr. Insul., vol. 1, no. 5, pp. 812-825, 1994.

[2] C. W. Reed, "Functionalization of nanocomposite dielectrics," Conf. Rec. IEEE Int. Symp. Electr. Insul., pp. 0-3, 2010.

[3] Y. Lin, T. Williams, and J. Connell, "Soluble, exfoliated hexagonal boron nitride nanosheets," J. Phys. Chem. Lett., vol. 1, no. 1, pp. 277-283, Jan. 2010

[4] I. L. Hosier, M. Praeger, a S. Vaughan, and S. G. Swingler, "Electrical properties of polymer nano-composites based on oxide and nitride fillers," 2015 IEEE Electr. Insul. Conf., no. June, pp. 438-441, 2015.

[5] K. Y. Lau, A. S. Vaughan, G. Chen, and I. L. Hosier, "Dielectric response of polyethylene nanocomposites: The effect of surface treatment and water absorption," 2012 Annu. Rep. Conf. Electr. Insul. Dielectr. Phenom., pp. 275-278, Oct. 2012.

[6] I. L. Hosier, M. Praeger, a F. Holt, a S. Vaughan, and S. G. Swingler, "Effect of water absorption on dielectric properties of nano-silica / polyethylene composites," 2014 Annu. Rep. Conf. Electr. Insul. Dielectr. Phenoma, pp. 651-654, 2014.

[7] D. Fabiani, G. Montanari, and L. Testa, "Effect of aspect ratio and water contamination on the electric properties of nanostructured insulating materials," IEEE Trans. Dielectr. Electr. Insul., vol. 17, no. 1, pp. 221-230, 2010.

[8] M. Praeger, I. L. Hosier, a S. Vaughan, and S. G. Swingler, "The effects of surface hydroxyl groups in polyethylene-silica nanocomposites," 2015 IEEE Electr. Insul. Conf., no. June, pp. 201-204, 2015. 